\title{
Mori-Zwanzig-Daubechies decomposition of Ising-model Monte Carlo dynamics
}

\author{
George D. J. Phillies \\ Jonathan J. Stott \\ Fairfield University, jstott@fairfield.edu
}

Follow this and additional works at: https://digitalcommons.fairfield.edu/physics-facultypubs

Copyright 1995 AIP Publishing

The final publisher PDF has been archived here with permission from the copyright holder.

\section{Peer Reviewed}

\section{Repository Citation}

Phillies, George D. J. and Stott, Jonathan J., "Mori-Zwanzig-Daubechies decomposition of Ising-model Monte Carlo dynamics" (1995). Physics Faculty Publications. 120.

https://digitalcommons.fairfield.edu/physics-facultypubs/120

\section{Published Citation}

Phillies, George DJ, and Jonathan Stott. "Mori-Zwanzig-Daubechies decomposition of Ising-model Monte Carlo dynamics." Computers in Physics 9, no. 2 (1995): 225-234.

This item has been accepted for inclusion in DigitalCommons@Fairfield by an authorized administrator of DigitalCommons@Fairfield. It is brought to you by DigitalCommons@Fairfield with permission from the rightsholder(s) and is protected by copyright and/or related rights. You are free to use this item in any way that is permitted by the copyright and related rights legislation that applies to your use. For other uses, you need to obtain permission from the rights-holder(s) directly, unless additional rights are indicated by a Creative Commons license in the record and/or on the work itself. For more information, please contact digitalcommons@fairfield.edu. 


\title{
Mori-Zwanzig-Daubechies decomposition of Ising-model Monte Carlo dynamics
}

\author{
George D. J. Phillies ${ }^{\mathrm{a})}$ \\ Department of Physics and Associated Biochemistry Faculty, Worcester Polytechnic Institute, \\ Worcester, Massachusetts 01609 \\ Jonathan Stott \\ Department of Physics, Case Western Reserve University, Cleveland, Ohio 44106
}

(Received 29 August 1994; accepted 4 January 1995)

\begin{abstract}
Monte Carlo dynamics of one- and two-dimensional Ising lattices were studied by computer simulation. A comparison of decompositions made with Haar and Daubechies wavelets finds that wavelet-wavelet time correlation functions $\left\langle c^{n}(t) c^{n}(t+\tau)\right\rangle$ and their long-time decay constants $\Gamma_{n}$ are virtually independent of the choice of wavelet basis. An intermediate-temperature scaling relation between $\Gamma_{n}$ and $\left\langle c^{n}(t) c^{n}(t+\tau)\right\rangle$ fails at low temperature. The temperature at which failure occurs decreases with increasing wavelet decimation level $n$. Mori-Zwanzig memory kernels $\phi(\tau)$ are extracted from $\left\langle c^{n}(t) c^{n}(t+\tau)\right\rangle$ without resort to Laplace transforms. Numerically, $\phi(\tau)$ computed from the random force autocorrelation function $\left\langle f_{i}(t) f_{i}(t+\tau)\right\rangle$ is in good agreement with $\phi(\tau)$ computed from the $\left\langle c^{n}(t) c^{n}(t+\tau)\right\rangle$. Even for a system as simple as the two-dimensional periodic Ising lattice with nearest-neighbor interactions, $\phi(\tau)$ is nonexponential; our results are consistent with a power-law decay of $\phi(\tau)$ at large $\tau$. (C) 1995 American Institute of Physics.
\end{abstract}

\section{INTRODUCTION}

The Ising ${ }^{1}$ model of a magnetic system has been a major topic of study since 1925 , when Ising studied properties of a one-dimensional ring of spins with nearest-neighbor interactions. More recently, statistico-mechanical properties of the Ising lattice have been a significant focus for investigation via computer simulation. The objective in this article is to demonstrate how certain new collective variables, namely the wavelet components treated by Strang ${ }^{2}$ and Daubechies, ${ }^{3}$ provide a useful description of Ising model dynamics. Wavelets are not widely familiar in physics or chemistry; in addition to Daubechies' work, ${ }^{3}$ Chui, ${ }^{4}$ and Kaiser $^{5}$ provide extended pedagogical discussions on wavelets.

We have previously examined ${ }^{6}$ the utility of applying to the one-dimensional Ising ring the Burt-Adelson ${ }^{7}$ wavelets sometimes used in image analysis. Here we explore the importance of the choice of wavelet basis: by varying the probe wavelets, can one get usefully different types of information about a system? Relative to our previous article, ${ }^{6}$ we obtain results over a wider range of temperatures, and cover two- as well as one-dimensional systems.

We further explore how wavelet-wavelet time correlation functions can be used to enhance a Langevin-like description of spin dynamics. For a collective variable $A$, a generalized Langevin equation may be written as

$$
\frac{d A(t)}{d t}=-\int_{0}^{t} d s A(s) \phi(t-s)+f_{i}(t),
$$

where $\phi(t-s)$ and $f_{i}(t)$ are the memory kernel and the

Author to whom correspondence should be addressed. E-mail: phillies@ wpi.wpi.edu (Internet). random force, respectively. In the familiar Langevin equation for a Brownian particle, $\phi(t-s)$ is proportional to a Dirac delta function $\delta(t-s)$. The fluctuation-dissipation theorem links the time evolutions of $f_{i}(t)$ and $\phi(t)$.

Mori ${ }^{8}$ and Zwanzig $^{9}$ have shown that in any system whose dynamics follow Lagrange's equation of motion the dynamics can be reduced via a projection operator formalism to equations having the form of Eq. (1). The significance of the Mori-Zwanzig formalism is that it actually provides a general way to extract exact Langevin-like equations, for a set of (typically collective) variables, from the complete Lagrangian. The Mori-Zwanzig formalism is perhaps more familiar to theoretical chemists ${ }^{10,11}$ than to theoretical physicists, so its core results are briefly noted below.

For the systems treated here, we applied Eq. (1) to analyze the dynamics of spin and wavelet (collective spin) variables. A procedure for extracting $\phi(t)$ from spin-spin and wavelet-wavelet correlation functions is given and applied. By applying the extracted $\phi(t)$ to a further simulation on the same system, we were able to obtain the random force $f_{i}(t)$ and show that the random force and memory kernel that we determine are consistent with the fluctuation-dissipation theorem, confirming the numerical validity of our $\phi(t)$.

The following sections treat wavelet projections, Ising models and Monte Carlo dynamics, an exploration of the significance of the wavelet basis in one-dimensional systems, dynamic scaling behavior of wavelet amplitude relaxations in two-dimensional systems, and the MoriZwanzig-Daubechies decomposition of wavelet dynamics in one- and two-dimensional systems. A discussion closes the article. 


\section{WAVEET PROJEETIONS}

Wavelets are members of the family of functions $h_{a, b}$ in which

$$
h_{a, b}(x)=|a|^{-1 / 2} h\left(\frac{x-b}{a}\right) .
$$

Wavelets are thus self-similar under a group of translations and dilations. $x$ may be continuous or discrete. In general, there are no restraints on the parameters $a$ or $b$; we consider applications in which $a=2$ and $b=1$. The resulting form

$$
H_{m, n}(x)=2^{-n / 2} h\left(2^{-n} x-m\right)
$$

for integer $m$ and $n$ may be used to generate wavelet families. Here, $m$ determines the translation and $n$ determines the order of the dilation. If $x$ is discrete rather than continuous, the $2^{-n}$ quantities refer to decimation rather than dilation.

Wavelets are of interest because they provide localized, orthogonal or nonorthogonal, complete or overcomplete sets of basis functions. Just as a function $F(t)$ may be written as a sum of Fourier functions $\cos \left(\omega_{n} t\right)$ and Fourier amplitudes $\hat{F}(n)$ as $\Sigma_{n} \hat{F}(n) \cos \left(\omega_{n} t\right)$, so also may a function be expanded as a sum of wavelets multiplied by wavelet amplitudes $c_{i}^{n}$ and $d_{i}^{n}$.

Wavelet components of a discrete function $s_{i}$ are obtained by wavelet transforms. A wavelet transform can generally be expressed as the sum of a linear low-pass filter $L$ and a linear high-pass filter $H$. The $L$ filter performs decimation and translation, while the $H$ filter recovers detail lost by $L .{ }^{2} L$ and $H$ jointly transform $N$ terms of $s_{i}$ into $N / 2$ smoothed terms $c_{i}^{1}$ and $N / 2$ high-frequency terms $d_{i}^{1}$. The transformation process is iterative, application of $L$ and $H$ to the $c_{i}^{j}$ yielding smoothed terms $c_{i}^{j+1}$ and high-frequency terms $d_{i}^{j+1}$. Here the subscript denotes the translational position of the wavelet and the superscript indicates the order of the decimation. Discrete wavelet transforms may be written as matrix multiplications. ${ }^{12}$

Work here is centered on an analysis of wavelet amplitudes. We used two types of wavelet families, namely the Haar wavelets and the Daubechies ${ }^{3}$ wavelets. One- or twodimensional spin patterns $s_{i}$ or $s_{i, j}$ were decomposed into smoothed and high-frequency wavelet components. Static and dynamic correlation functions of the $c_{i}^{n}$ and $d_{i}^{n}$ were then computed, gaining information about equilibrium and time-dependent properties of Ising lattices.

The continuum Haar wavelets have as a basis function ${ }^{3}$

$$
h(x)=\left\{\begin{array}{cc}
1, & 0 \leqslant x<0.5 \\
-1, & 0.5 \leqslant x<1 \\
0, & \text { otherwise }
\end{array}\right.
$$

The remainder of the Haar wavelets are generated from $h(x)$ by application of Eq. (3). For a discrete system, Eq. (4) is replaced by

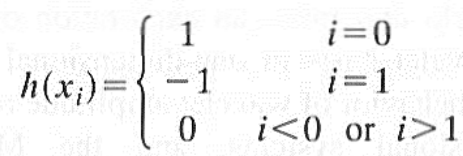

Discrete Haar wavelets are orthogonal, in that a scalar product of two Haar wavelets obtained from Eqs. (3) and (5) can be written

$$
\sum_{n} \sum_{n^{\prime}} 2^{-m / 2} h\left(2^{-m} x-n\right) 2^{-m^{\prime} / 2}\left(2^{-m^{\prime}} x-n^{\prime}\right)=\delta_{m m^{\prime}}
$$

For the discrete Haar wavelets, Strang ${ }^{2}$ derives fundamental filters $L$ and $H$

$$
\begin{aligned}
& L=\left(\begin{array}{ll}
1 & 1
\end{array}\right), \\
& H=\left(\begin{array}{ll}
1 & -1
\end{array}\right) .
\end{aligned}
$$

Filters act by vector multiplication on an $\mathrm{N}$-component data vector $\left\{s_{i}\right\} . L$ and $H$ may be combined into one matrix operation, the complete $N \times N$ transformation matrix being

$$
\mathbf{D}_{\mathbf{H}}=\left(\begin{array}{ccccc}
1 & 1 & 0 & 0 & \\
1 & -1 & 0 & 0 & \ldots \\
0 & 0 & 1 & 1 & \\
0 & 0 & 1 & -1 & \\
& & \vdots & & \ddots
\end{array}\right)
$$

Applying the decomposition matrix $D_{H}$ to a vector $S$ containing an even number of components yields a new vector whose elements are alternately the low-frequency components $c_{i}^{1}$ and the high-frequency components $d_{i}^{1}$, i.e.,

$$
\mathbf{D}_{\mathrm{H}} \mathbf{S}=\left\{c_{0}^{1}, d_{0}^{1}, c_{1}^{1}, d_{1}^{1}, \ldots, c_{-1+n / 2}^{1}, d_{-1+n / 2}^{1}\right\} .
$$

$\mathbf{D}_{\mathrm{H}} \mathbf{S}$ is usefully permuted as

$$
\mathbf{S}_{\mathbf{P}}=\left\{c_{0}^{1}, c_{1}^{1}, \ldots c_{-1+n / 2}^{1}, d_{0}^{1}, d_{1}^{1}, \ldots d_{-1+n / 2}^{1}\right\} .
$$

Recursive application of an $N / 2 \times N / 2$ component of $\mathbf{D}_{\mathrm{H}}$ to the $c_{i}^{n-1}$ yields higher-order decompositions $c_{i}^{n}$ and $d_{i}^{n}$. Note that the decimation process is intrinsically recursive; each order $n$ of wavelet components has half as many terms as the previous order did.

The Haar basis ${ }^{3}$ is the only wavelet basis which is orthonormal, has compact support, and has a symmetry axis. Daubechies ${ }^{3}$ has developed a family of orthonormal wavelets which are compactly supported but lack a reflection axis. For the discrete case these wavelets bases are labeled by the number of their components as $D_{2 N}$, e.g., $D_{4}, D_{6}, \ldots$; Daubechies ${ }^{3}$ tabulates coefficients equivalent to Eq. (5) for $N=2, \ldots, 10$. High and low pass filters for $D_{4}$ are $^{3}$

$$
\begin{aligned}
& L_{D 4}=\left(\begin{array}{llll}
c_{0} & c_{1} & c_{2} & c_{3}
\end{array}\right), \\
& H_{D 4}=\left(\begin{array}{llll}
c_{3}-c_{2} & c_{1} & -c_{0}
\end{array}\right),
\end{aligned}
$$

where $c_{0}=(1+\sqrt{3}) / 4 \sqrt{2}, c_{1}=(3+\sqrt{3}) / 4 \sqrt{2}, c_{2}=(3-\sqrt{3}) / 4$ $\sqrt{2}$, and $c_{3}=(1-\sqrt{3}) / 4 \sqrt{2}$.

Some of the following simulations were made on two dimensional Ising lattices. A two-dimensional wavelet transformation of a square lattice may be obtained by making a one-dimensional transformation and permutation of each row of the lattice, so that individual $s_{i, j}$ are replaced with $\left(c_{i}^{1}\right)_{j}$ and $\left(d_{i}^{1}\right)_{j}$ as shown in Eq. (10). The notation $(\cdots)_{j}$ refers to the contents of the $j$ th row of the lattice after each row has separately been wavelet-decomposed via $L$ and $H$, and then permuted. The $\left(c_{i}^{1}\right)_{j}$ and $\left(d_{i}^{1}\right)_{j}$ form a square lattice, with one component to each column. A onedimensional transformation on each column of the transformed matrix then yields the two-dimensional wavelet transformation of the matrix. The components of the transformed matrix may formally be described as arising from $L \otimes L, L \otimes H, H \otimes L$, and $H \otimes H$. Iterative application of the 
filters, alternately to rows and to columns, yields all higherorder two-dimensional wavelet components.

\section{ISING MODE}

This article treats simulations of one- and two-dimensional Ising lattices with nearest neighbor interactions. The Ising model Hamiltonian for the one-dimensional lattice was written

$$
H=\sum_{i=1}^{N} J s_{i} s_{i+1},
$$

where for $N$ spins and periodic boundary conditions $s_{N+j}=s_{j}$. Here $s_{i}= \pm 1$ is constrained. On a twodimensional $L \times L$ lattice with periodic boundary conditions, the Hamiltonian was

$$
H=\sum_{i=1}^{L} \sum_{j=1}^{L} J\left(s_{i, j} s_{i+1, j}+s_{i, j} s_{i, j+1}\right),
$$

where $s_{i, j}$ [the spin at the $(i, j)$ lattice site] has allowed values \pm 1 , and where periodic boundary conditions give $s_{i, j}=s_{i, j+N}$ and $s_{i, j}=s_{i+N, j}$.

Static and dynamic properties of the one-dimensional Ising ring were treated analytically by Glauber, ${ }^{13}$ who showed that the single-time spin-spin correlation function is

$$
\left\langle s_{i}(t) s_{i+n}(t)\right\rangle=\eta^{n},
$$

where $s_{i}(t)$ is the value of the $i$ th spin at time $t$ and where $\eta=\tanh (\beta J)$ is a short-range order parameter. ${ }^{13}$ Glauber also presents two-time spin-spin correlation functions for a dynamics not quite the same as ours. From Eq. (14) and the wavelet definitions, analytic calculations of mean-square wavelet amplitudes $\left\langle\left[c_{i}^{n}(t)\right]^{2}\right\rangle$ and $\left\langle\left[d_{i}^{n}(t)\right]^{2}\right\rangle$ are possible. The system Hamiltonian only determines the equilibrium (static) properties of the spin lattices. Spin dynamics were taken to arise from interactions of the spins with a virtual heat bath not represented in Eqs. (12) and (13).

We treat Ising lattice dynamics by computer simulation using the Metropolis algorithm, ${ }^{14}$ which describes spins with internal interactions given by Eqs. (12) or (13) and external interactions with a heat bath. The computer models the bath interactions by randomly selecting a spin and computing the change $\Delta H$ in the Hamiltonian attendant to flipping that spin. If $\beta \Delta H<0$, the spin is flipped, where $\beta=\left(k_{B} T\right)^{-1}$ is the reciprocal temperature in energy units. If $\beta \Delta H>0$, the spin is flipped with probability $P=\exp (-\beta \Delta H)$ on comparison of $P$ with a computergenerated pseudorandom number. To ascribe a dynamics to this system, the notional time variable is the number of attempted spin flips, whether successful or not, in natural units of flips per spin.

Note that Metropolis-Monte Carlo dynamics are not the same as the dynamics treated in Ref. 13. While both types of dynamics satisfy the principle of detailed balance, the principle of detailed balance controls the ratio of reactions rates for flipping a spin up or down, not the absolute rate for either process. We therefore only make quantitative comparisons with Ref. 13's equilibrium results.

Simulations on one-dimensional systems used primarily 1024-spin rings; control simulations made with rings containing 256 to 2048 spins showed no sensitivity of our results to ring size. Two-dimensional simulations were largely made on $64 \times 64$ square lattices. One-dimensional simulations on cold systems (e.g., $\beta J=2.0$ ) involved 400000 attempted flips per spin, shorter simulations being found adequate at higher temperatures. Two-dimensional simulations on warmer systems $(\beta J \leqslant 0.6)$ extended over shorter times, typically $10000-50000$ attempted flips per spin.

Each simulation was preceded by a thermalization process adequately long to equilibrate all spins. The simulations had four fundamental parts, namely operation of the spin dynamics, projection of the spins into their wavelet components, computation of wavelet-wavelet correlation functions, and analysis of the correlation functions. Wavelet projections and correlation functions were computed on the fly; correlation functions were stored and analyzed post facto. Based on our previous work, ${ }^{6}$ we focused primarily

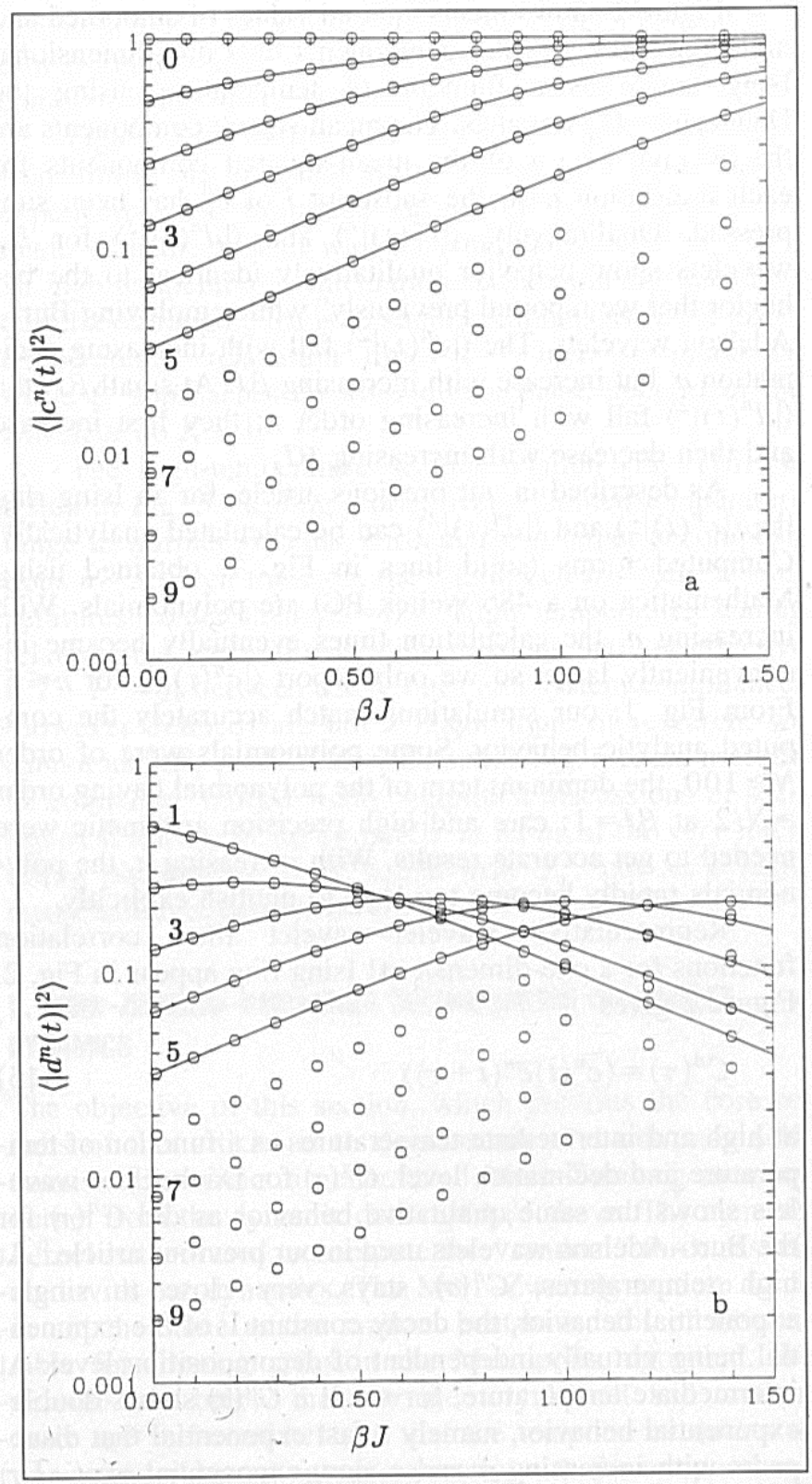

Figure 1. Single time correlation functions $(a)\left\langle\left|c^{n}(t)\right|^{2}\right\rangle$ and $(b)$ $\left\langle\left|d^{n}(t)\right|^{2}\right\rangle$ of Daubechies $D_{4}$ wavelet amplitudes as a function of inverse temperature $\beta J$ for wavelets of order $n=0-9$ (labels 0, 3, 5, 7, 9 denote decimations $n$ ). The underlying Ising lattice is a one-dimensional 1024spin ring. Solid lines represent analytic calculations for fixed $n$. 
on smoothed wavelets, calculating single-time and twotime correlation functions for various wavelet bases and decimation levels $n$.

\section{ONE-DIMENSIONAL SYSTEMS: SIGNIFICANCE OF THE WAVEFT BASIS}

The literature contains a very wide range of wavelet bases, including the Haar and Daubechies wavelets described above and the Burt-Adelson basis ${ }^{7}$ employed in our previous article. ${ }^{6}$ Clearly, if each choice of basis emphasized substantially different aspects of the same fundamental data, selecting the basis would become a fundamental question in applying wavelet projections to physical problems. To study the importance of various wavelet bases, we calculated the static and dynamic properties of the Ising ring for Haar wavelets and for Daubechies wavelets $D_{2 N}$ for $N$ of 2-5.

Figure 1 shows mean-squared values of smoothed and high-frequency wavelet components of a one-dimensional Ising lattice as a function of temperature, using the Daubechies $D_{4}$ wavelets. The mean-square components are the average over $i$ of the mean-squared components for each translation $i$, so the subscript $i$ of $c_{i}^{n}$ has been suppressed. Qualitatively, $\left\langle\left|c^{n}(t)\right|^{2}\right\rangle$ and $\left\langle\left|d^{n}(t)\right|^{2}\right\rangle$ for $D_{4}$ wavelets show behavior qualitatively identical to the behavior that we reported previously ${ }^{6}$ while employing BurtAdelson wavelets. The $\left\langle\left|c^{n}(t)\right|^{2}\right\rangle$ fall with increasing decimation $n$, but increase with increasing $\beta J$. At small $\beta J$, the $\left\langle\left|d^{n}(t)\right|^{2}\right\rangle$ fall with increasing order $n$; they first increase and then decrease with increasing $\beta J$.

As described in our previous article, for an Ising ring the $\left\langle\left|c^{n}(t)\right|^{2}\right\rangle$ and $\left\langle\left|d^{n}(t)\right|^{2}\right\rangle$ can be calculated analytically. Computed forms (solid lines in Fig. 1, obtained using Mathematica on a $486 /$ Weitek PC) are polynomials. With increasing $n$, the calculation times eventually became inconveniently large, so we only report $\left\langle\left|c^{n}(t)\right|^{2}\right\rangle$ for $n \leqslant 5$. From Fig. 1, our simulations match accurately the computed analytic behavior. Some polynomials were of order $N \geqslant 100$, the dominant term of the polynomial having order $\approx N / 2$ at $\beta J=1$; care and high precision arithmetic were needed to get accurate results. With increasing $n$, the polynomials rapidly become too long to publish explicitly.

Representative wavelet-wavelet time correlation functions for a one-dimensional Ising ring appear in Fig. 2. Figure 2 gives

$$
C^{n}(\tau)=\left\langle c^{n}(t) c^{n}(t+\tau)\right\rangle
$$

at high and intermediate temperatures as a function of temperature and decimation level. $C^{n}(\tau)$ for Daubechies wavelets shows the same qualitative behavior as $\operatorname{did} C^{n}(\tau)$ for the Burt-Adelson wavelets used in our previous article. ${ }^{6} \mathrm{At}$ high temperatures, $C^{n}(\tau)$ stays very close to singleexponential behavior, the decay constant $\Gamma$ of the exponential being virtually independent of decomposition level. At intermediate temperature, for small $n C^{n}(\tau)$ shows doubleexponential behavior, namely a fast exponential that disappears with increasing $n$ and a slow exponential $\exp (-\Gamma \tau)$ whose $\Gamma$, extracted by nonlinear fitting techniques, is nearly independent of $n$.

$C^{n}(\tau)$ was computed for Haar wavelets and for $D_{2 n}$ wavelets for $n \in(2,5)$. Figure 2 shows sample outcomes. For clarity, only Haar, $D_{4}$ and $D_{10}$ wavelets are plotted. At fixed $n$ and $\tau, C^{n}(\tau)$ is virtually independent of the wavelet

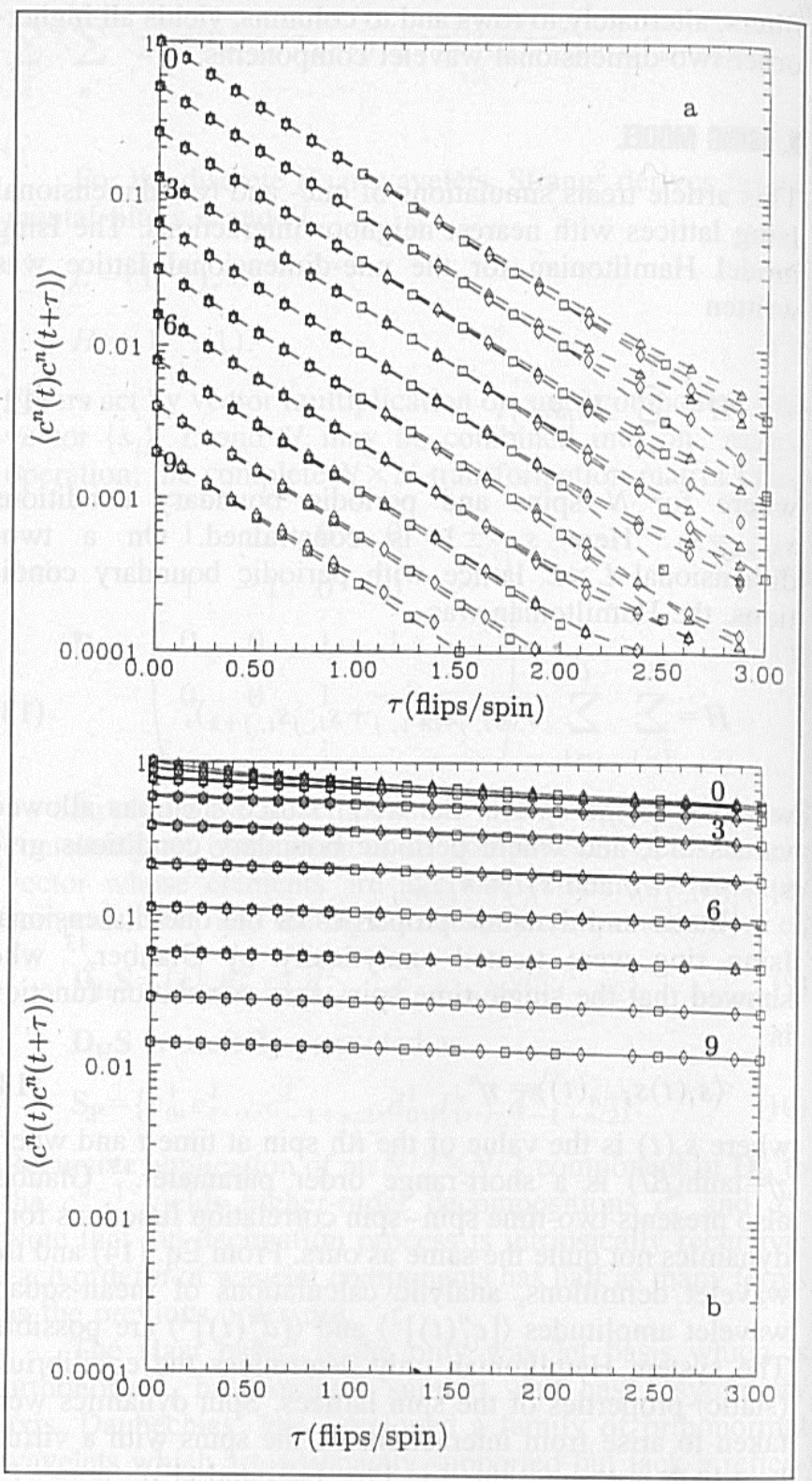

Figure 2. Comparison of Haar (diamonds), Daubechies $D_{4}$ (squares), and Daubechies $D_{10}$ (triangles) wavelets using a one-dimensional Ising ring. $n$ (labels mark $n=0,3,6,9)$. Wavelet-wavelet time correlation functions $\left\langle c^{n}(t) c^{n}(t+\tau)\right\rangle$ at (a) high $(\beta J=0.1)$ and (b) intermediate $(\beta J=1.0)$ temperatures are plotted against delay time $\tau$.

basis. The remainder of this article therefore focuses largely on computations using a single wavelet species, namely the $D_{4}$ wavelets.

A comparison of $\Gamma$ with the system compliance $\left\langle\left|c^{n}(t)\right|^{2}\right\rangle$ provides a test of scaling relationships

$$
\Gamma=\left\langle\left|c^{n}(t)\right|^{2}\right\rangle^{-z / 2},
$$

where $z$ is a scaling exponent. In the previous article, ${ }^{6}$ we tested this relationship for Burt-Adelson ${ }^{7}$ wavelets, finding $z \approx 1.8$. Equation $(16)$ refers to the decay of all fluctuations within the support of a given wavelet, not to the decay of fluctuations of some mean size $\xi$, so arguments predicting relaxation times $\Gamma^{-1}$ that scale as $\xi^{2}$ do not apply in unmodified form to our relaxation times. The previous article ${ }^{6}$ predicted that Eq. (16) can only be valid at intermediate 


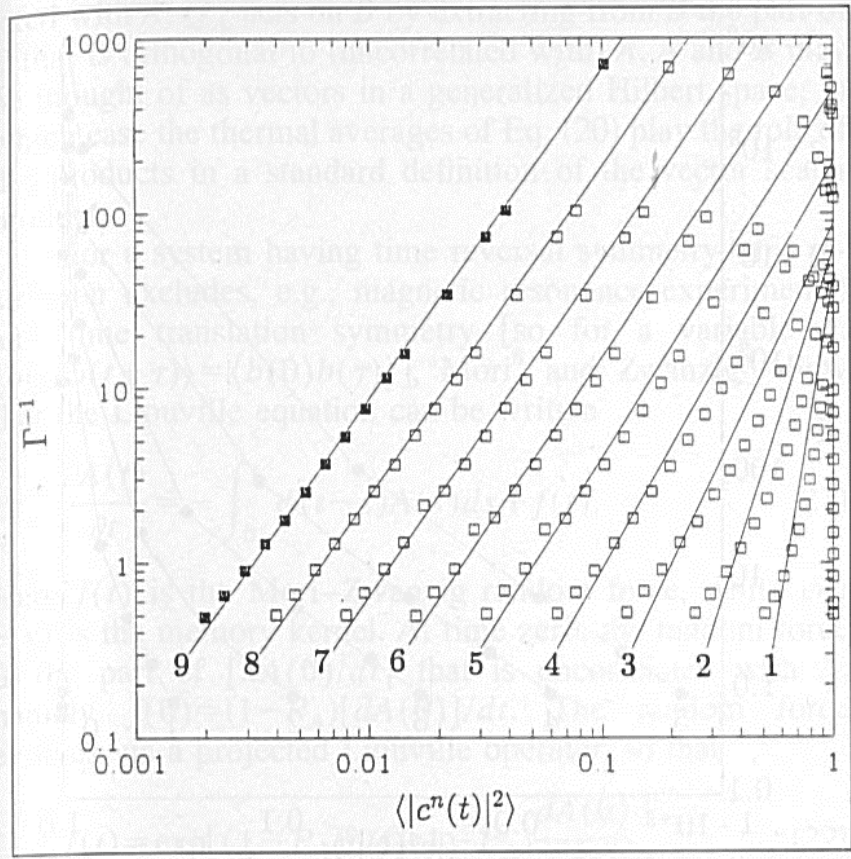

Figure 3. Long-time exponential decay time $\Gamma^{-1}$ against mean-square amplitude $\left\langle\left|c^{n}(t)\right|^{2}\right\rangle$ of $D_{4}$ wavelets for a one-dimensional Ising ring, testing intermediate-temperature scaling $\left(\Gamma^{-1}=\left(\left\langle\left|c^{n}(t)\right|^{2}\right\rangle\right)^{z / 2}\right.$; straight lines). Lines connect points of equal $n$; filled squares denote $n=9$ with other $n$ labeled. Here $\beta J \in(0,2.5) ; \beta J$ increases towards the top of the graph.

temperatures. In the limit $T \rightarrow 0$, one expects $\Gamma \rightarrow 0$ but $\left\langle\left|c^{n}(t)\right|^{2}\right\rangle \rightarrow 1$, so Eq. (16) necessarily fails in the lowtemperature limit.

Figure 3 shows an extensive test of Eq. (16) at temperatures $\beta J \in(0,2.5)$. Figure 3 plots $\Gamma^{-1}$ against $\left\langle\left|c^{n}(t)\right|^{2}\right\rangle$. Displayed data refer to $D_{4}$ wavelets; data based on other Daubechies wavelets can be superposed on the points seen here. The decay constant falls nearly 1000 -fold over the temperature interval that we studied. In Fig. 3, lines unify points of equal decomposition $n$ at a series of temperatures. Filled squares refer to $n=9$; points corresponding to single spins $(n=0)$ lie on the right-hand vertical coordinate axis. Points of highest temperature are at the bottom of the graph; temperature falls as one moves upwards.

Figure 3 covers a considerably wider range of $n$ and $T$ than our previous analysis. Low-decimation data points (right-hand side of Fig. 3), previously found to lie on nearly straight lines, obviously curve away from power-law behavior at low temperatures, as we had predicted. The degree of deviation decreases with increasing $n$. At the highest decomposition level that we examined, Eq. (16) works well over three orders of magnitude in $\Gamma^{-1}$. There is at most a slight dependence of $z$ on the choice of Daubechies wavelet; we consistently find $z$ in the range $1.73-1.81$ for $D_{4}, D_{6}, D_{8}$, and $D_{10}$ wavelets.

\section{TWO-DIMENSIONAL SIMULATIONS}

While the Ising ring's properties have been found analytically, albeit not for our dynamics, the analytic behavior of the two-dimensional Ising lattice remains an active topic of investigation. This section examines wavelet decompositions of a two-dimensional $64 \times 64$ square lattice with periodic boundary conditions. In two dimensions, a wavelet decomposition involves sequential decomposition into smoothed and high-frequency components along alternate axes. At each level $n$ the decomposition yields four sets of wavelet components, corresponding to smoothing or highfrequency extraction along each axis. Here we focus on wavelet components $c^{n}(t)$ that have been smoothed along both axes.

Figure 4 presents representative wavelet-wavelet time correlation functions, as functions of $\tau$ and $n$, at the moderately high temperature $\beta J=0.3$. For a $64 \times 64$ lattice and $D_{4}$ wavelets we are limited to $n \leqslant 5$. For larger $n$, the support of the wavelet would be wider than 64 spins, so that (given the periodic boundary conditions), the support would wrap around and overlap itself. The zero-time correlation functions $\left\langle\left|c^{n}(t)\right|^{2}\right\rangle$ depend inversely on $n$. At larger $n,\left\langle c^{n}(t) c^{n}(t+\tau)\right\rangle$ decays nearly exponentially in $\tau$. For $n \leqslant 3$ the decay is markedly nonexponential, with a rapid early decay and a long time nearly exponential decay. In one dimension, at large $\tau$ a plot of the $\left\langle c^{n}(t) c^{n}(t+\tau)\right\rangle$ reveals a series of near-parallel lines. In contrast, in two dimensions at large $\tau$ the $\left\langle c^{n}(t) c^{n}(t+\tau)\right\rangle$ converge to a common value that slowly decays.

Figure 5 gives the slow decay times $\Gamma^{-1}$ extracted from fitting the long-time decay of $\left\langle c^{n}(t) c^{n}(t+\tau)\right\rangle$ to an exponential. We examined $\beta J \in(0.1,0.6)$. Comparison is made with the system wavelet compliance $K=\left\langle\left|c^{n}(t)\right|^{2}\right\rangle$. At high temperatures, fluctuations decay quickly; the short correlation range $\xi$ leads to small compliances $K$. At lower temperatures, relaxation times $\Gamma^{-1}$ increase markedly, while $\xi^{2}$ approaches the (two-dimensional) support of the wavelets, so $K \rightarrow 1$.

The high-temperature scaling relation [Eq. (16)] is tested in Fig. 5. Scaling works over a limited temperature range in warmer systems, particularly at larger decompositions $n \geqslant 3$. Even for $n=5$, the relation clearly fails at temperatures colder than $\beta J=0.4$. High temperature scaling relations (as opposed to scaling relations that are effective if $T-T_{c} \rightarrow 0$ ) between decay rates and system compliances (however defined) are not a major topic of research; we cannot identify predictions with which Fig. 5 could usefully be compared. Indeed, many simplified discussions of scaling treat scaling behavior purely in terms of the very interesting phenomena to be found near $T_{c}$, not as a hightemperature leading behavior.

\section{MORI-ZWANZG-DAUBECHIES DECOMPOSITION OF WAVEFT DYNAMICS}

The objective of this section, which presents the core results of this article, is to demonstrate that one may selfconsistently extract a generalized Mori-Zwanzig memory kernel from the dynamic data reported above. The memory kernel is then used to compute the "random" force associated with our dynamics. The Mori-Zwanzig random force is not the same as the random heat-bath "force" responsible for flipping spins in the Metropolis algorithm. We demonstrate below that our memory kernel and random force are self-consistent, in that the temporal evolution of our memory kernel agrees correctly with the temporal autocorrelation function of the random force computed while using our memory kernel.

The Mori-Zwanzig formalism ${ }^{8,9}$ may not be uniformly familiar to all readers. We first sketch its principal results, which were obtained for systems whose complete Hamiltonians were known, and then show how the results 


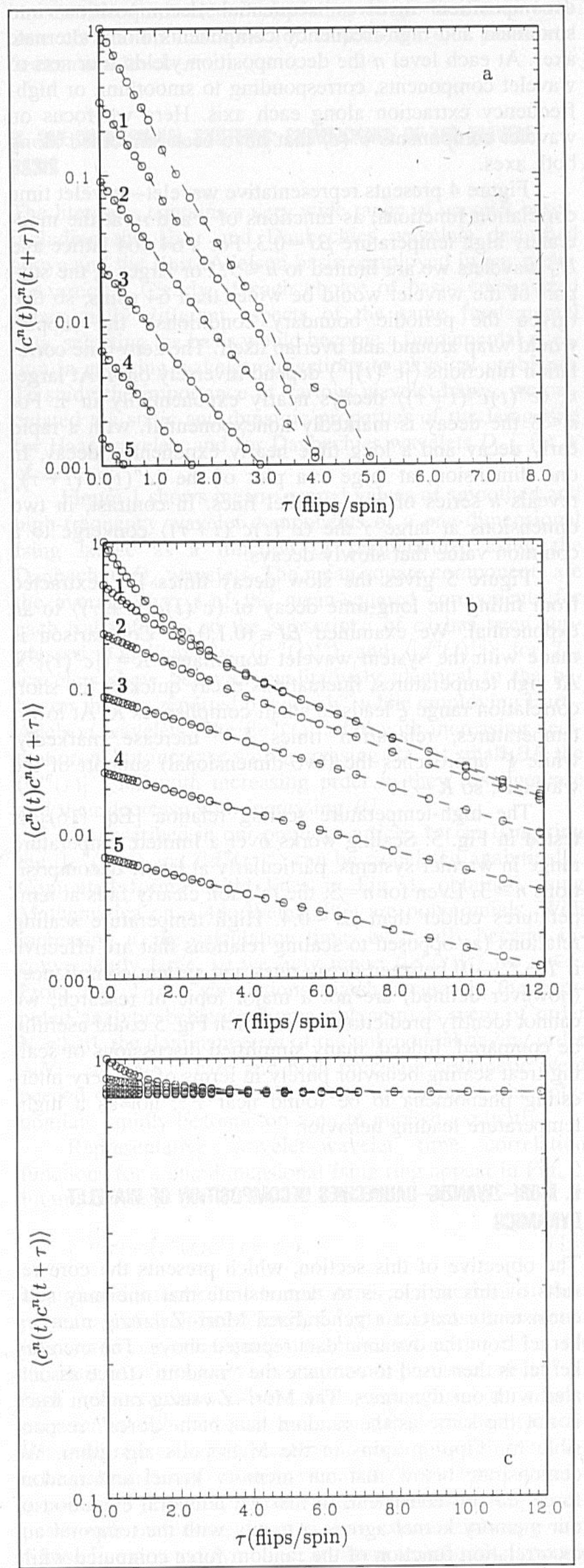

Figure 4. Wavelet-wavelet time correlation functions $\left\langle c^{n}(t) c^{n}(t+\tau)\right\rangle$ for a $64 \times 64$ periodic Ising lattice using smoothed components of $D_{4} \otimes D_{4}$ wavelets at temperatures $\beta J$ of (a) 0.1 , (b) 0.3 , and (c) 0.5. Decomposition levels $n \in(0,5)$ are labeled in (a) and (b), but are indistinguishable at larger $\tau$ in $(c)$.

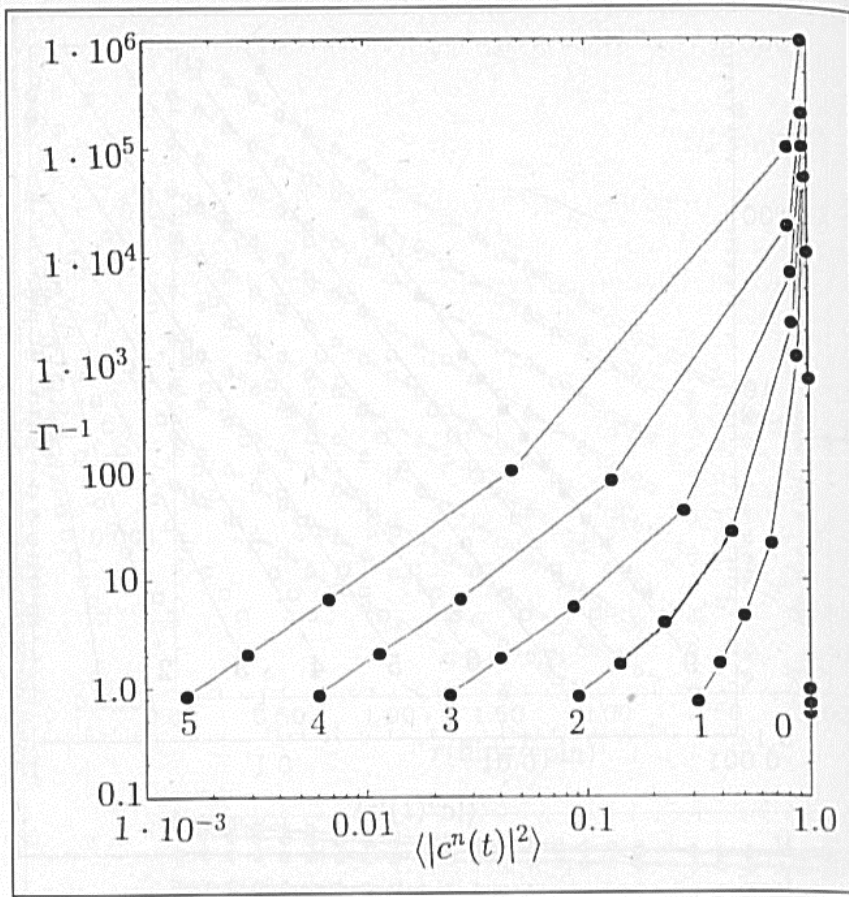

Figure 5. Long-time exponential decay time $\Gamma^{-1}$ against mean-square wavelet amplitude for a square Ising lattice decomposed via $D_{4} \otimes D_{4}$ smoothed wavelets. Lines connect points of equal $n$ (labeled on graph); $T$ falls ( $\beta J$ increases) towards the top of the graph, which covers $\beta J \in(0,0.6)$.

can be applied to systems having Metropolis-Monte Carlo dynamics. The Mori-Zwanzig formalism provides a systematic method for proceeding from the Liouville equation

$$
\frac{d A}{d t}=\mathscr{L} A
$$

for the time evolution of a variable $A$ to a Langevin-like equation for the same variable. Formally, Eq. (17) has the solution

$$
A(t)=\exp (t \mathscr{L}) A(0) .
$$

Here

$$
\mathscr{L}=\sum_{i=1}^{N} \frac{\partial H}{\partial p_{i}} \frac{\partial}{\partial r_{i}}-\frac{\partial H \partial}{\partial r_{i} \partial p_{i}}
$$

is the Liouville operator, where $H$ is the Hamiltonian, and where the $N$ pairs of variables $\left(r_{i}, p_{i}\right)$ are positions and canonically conjugate momenta. The dynamic variable $A$ may be one of the $r_{i}$ or $p_{i}$ or a function of those variables; in the extended form of the formalism $A$ may be a vector (an ordered list) of mechanical variables.

Fundamental to the Mori-Zwanzig theory is the projection operator $P_{A}$, where for a variable $B$ the projected variable $P_{A}(B)$ is

$$
P_{A}[B(t)]=\frac{\langle A(t) B(t)\rangle A(t)}{\left\langle[A(t)]^{2}\right\rangle}
$$

The brackets $\langle\cdots\rangle$ denote a conventional thermal average; Eq. (20) is to be modified if (a case not relevant here) the dynamic variables are complex rather than real. Using 1 to represent the identity operator, $Q_{A}=1-P_{A} . P_{A}$ acts on a variable $B$ by extracting from it the part of $B$ that is corre- 
lated with $A ; Q_{A}$ acts on $B$ by extracting from $B$ the part of $B$ that is orthogonal to (uncorrelated with) $A$. $A$ and $B$ may be thought of as vectors in a generalized Hilbert space, in which case the thermal averages of Eq. (20) play the role of dot products in a standard definition of the/vector scalar product.

For a system having time reversal symmetry (this restriction excludes, e.g., magnetic resonance experiments) and time translation symmetry [so for a variable $b$, $\langle b(t) b(t+\tau)\rangle=\langle b(0) b(\tau)\rangle]$, Mori ${ }^{8}$ and Zwanzig ${ }^{9}$ show that the Liouville equation can be written

$$
\frac{\partial A(t)}{\partial t}=-\int_{0}^{t} \phi(t-s) A(s) d s+f(t) .
$$

Here $f(t)$ is the Mori-Zwanzig random force, while $\phi(t$ $-s)$ is the memory kernel. At time zero, the random force is the part of $[d A(0) / d t]$ that is uncorrelated with $A$, namely $f(0)=\left(1-P_{A}\right)[d A(0)] / d t$. The random force evolves via a projected Liouville operator, so that

$$
f(t)=\exp \left[\left(1-P_{A}\right) t \mathscr{L}\right]\left(1-P_{A}\right) \frac{d A(0)}{d t}
$$

The time evolution of $f(t)$ is to be contrasted with the time evolution of an orthodox mechanical variable, which evolves according to Eq. (18).

The memory kernel is determined by the autocorrelation function of the random force, namely

$$
\phi(t-s)=\frac{\langle f(t) f(s)\rangle}{\left\langle|A(0)|^{2}\right\rangle} .
$$

In the simplest case of Brownian motion governed by the Langevin equation, $f(t)$ has a correlation time of zero, while $\phi(t-s)$ is a Dirac delta function centered at $t-s$.

In typical applications of the formalism, ${ }^{15,16}$ the (perhaps collective) variable or variables with the slowest relaxation(s) are grouped into $A$, and various approximations are used to evaluate $\phi(t)$. Because the slowly relaxing variables have been projected out of the dynamics, $\phi(t)$ is commonly assumed to be short-lived and to have a relatively simple form. However, nothing in the derivation of the Mori-Zwanzig equation refers to the relative relaxation rates of the variables isolated by $P_{A}$ or by $Q_{A}$; the identification of $A$ with the "slow variable(s)" is a step of convenience that often leads to interesting results. It is almost always the case that an approximation scheme is used to obtain $\phi(t)$. There is rarely an explicit examination of the difference between normal and projected dynamics, though note Ref. 16, so that the detailed nature of projected dynamics remains an ill-studied part of statistical mechanics.

In the present case we do not have a complete Hamiltonian, so the system treated here is not an example of the Hamiltonian systems for which the Mori ${ }^{8}-\mathrm{Zwanzig}^{9}$ derivation was performed. However, that derivation uses only certain limited features of the Liouville operator. In particular, the derivation assumes that the system has a time translation operator $\mathscr{L}$ that propagates arbitrary mechanical variables $b$ via point contact transformations, the translation leading to time translation symmetry and (special case) stationarity (i.e., $\left.\left\langle[b(t)]^{2}\right\rangle=\left\langle[b(0)]^{2}\right\rangle\right)$. These requirements also constrain the averaging process $(\langle\cdots\rangle)$, but the derivation does not make explicit use of our identification of $\langle\cdots\rangle$ with a canonical ensemble average, in the sense that, e.g., the Yvon identity relies on the form of the canonical statistical weight. The Mori derivation of the Mori-Zwanzig equation is thus a formal statement about time evolution. The statement is formal in the sense that the derivation refers to the highly interesting case of a system whose complete Hamiltonian is known, but the derivation is actually valid for a much wider set of systems, including in particular the systems whose time evolutions are studied in this article.

In this section we use the formalism of Eqs. (21)-(23) to analyze the wavelet dynamics simulated above. The time evolution of our system is not governed by a known Hamiltonian, but the system satisfies the physical conditions needed for the Mori-Zwanzig arguments to be applicable. Since our time evolution operator uses a discrete rather than a continuous time variable, derivatives and integrals must be replaced with difference equations and summations. In particular, Eq. (21) is rewritten

$$
\frac{A_{i+1}-A_{i}}{t_{i+1}-t_{i}}=\sum_{j=0}^{i}\left(A_{j} \phi_{i-j}\right)+f_{i} .
$$

Here subscripts refer to discrete times labeled by $i$, so $A_{i}$ and $f_{i}$ are the dynamic variable of interest and the random force at time $i$, while $\phi_{i-j}$ is the memory function bridging times labeled by $i$ and $j$. The smallest identifiable time step in our simulation is a single attempted spin flip, but there is no fundamental reason not to treat a series containing several attempted spin flips as the fundamental dynamic unit. Natural time units here are attempted flips per spin, but $A$ can be sampled more or less frequently than once per natural time unit. $i$ and $t_{i}$ are therefore independent variables.

To determine $\phi_{i}$, one multiples Eq. (24) by $A_{0}$ and takes the thermal average, using the feature that the random force is uncorrelated with the $A_{j}$ at earlier times to set $\left\langle A_{0} f_{i}\right\rangle=0, i>0$. One writes

$$
\frac{\left\langle A_{0} A_{i+1}\right\rangle-\left\langle A_{0} A_{i}\right\rangle}{t_{i+1}-t_{i}}=\sum_{j=0}^{i} \phi_{i-j}\left\langle A_{j} A_{0}\right\rangle .
$$

When the autocorrelation functions $\left\langle A_{0} A_{i+1}\right\rangle$ are known quantities determined from Monte Carlo dynamics simulations, Eq. (25) implies a soluble series of coupled linear equations for the $\phi_{i}$, the first of which is

$$
\frac{\left\langle A_{0} A_{1}\right\rangle-\left\langle\left|A_{0}\right|^{2}\right\rangle}{t_{1}-t_{0}}=\phi_{0}\left\langle A_{0} A_{0}\right\rangle \text {. }
$$

On the other hand, if the memory function $\phi_{i}$ is known, Eq. (24) may be solved for the random force $f_{i}$, namely

$$
f_{i}=\frac{A_{i+1}-A_{i}}{t_{i+1}-t_{i}}-\sum_{j=-\infty}^{i} A_{j} \phi_{i-j},
$$

allowing determination of the true random force $f_{i}$ from a record of the $A_{i}$. Once the $f_{i}$ are calculated, one can perform a self-consistency check from an adequately converged set of $\phi(t)$ (i.e., a set taken out to sufficiently large $t$ ), by ascertaining whether or not the computed $f_{i}$ and the memory kernel satisfy Eq. (23). This self-consistency check should be expected to deteriorate as the time separation $t_{i+1}-t_{i}$ of the $\phi_{i}$ is increased.

We first tested this approach for obtaining $\phi_{i}$ by performing two independent simulations on the same Brownian particle driven by white noise. In the first run, from Eq. (25) we recovered the correct form for $\phi_{i}$, namely a delta- 
function spike whose measured amplitude agreed with amplitude calculated from the standard theory of Brownian motion. In the second run, the computed $\phi_{i}$ from the first run was used to calculate the random part $f_{i}$ of the driving force. The temporal autocorrelation function of $f_{i}$ was then obtained; $\phi_{j}$ and $\left\langle f_{i} f_{i+j}\right\rangle$ were in agreement with Eq. (23).

If $\phi_{j}$ and $\left\langle f_{i} f_{i+j}\right\rangle$ had been obtained from iterative analyses of a single simulation run, agreement with Eq. (23) would only mark satisfaction of a mathematical identity. The data sets we used to infer $\phi_{i}$ and $\left\langle f_{i} f_{j+i}\right\rangle$ were independent, so the noise terms in the two determinations were also independent, except that an error in measuring $\phi$ automatically leads to an error in calculating $f$. Differences between our $\phi_{i}$ and our $\left\langle f_{i} f_{j+i}\right\rangle$ arise from the noise in our stochastic determinations of these quantities; the difference between the two approaches to $\phi(t)$ represents the statistical sampling error in the simulations.

This method for finding $\phi$ and $f$ was then applied to the one- and two-dimensional Ising systems. A straightforward application of Eq. (25) to determine the $\phi_{i}$ requires that the $\left\langle A_{0} A_{i}\right\rangle$ be determined at points $t_{i}$ that are spaced uniformly in time. The simulations used to evaluate the $\phi_{i}$ were therefore not in all cases the same as the simulations discussed in the previous sections, because in some previous cases we computed $\left\langle A_{0} A_{i}\right\rangle$ at quasilogarithmically spaced times.

Figure 6 shows memory functions computed for the one-dimensional Ising ring at high and low temperatures. Here times $t$ are expressed in natural units. A separate calculation of $\phi(t)$ is required at each decimation $n$. The time variable was discretized in units of $1 / 8$ flip per spin. For each $n, \phi(t)$ has a spike at time zero, corresponding to the approximate exponential decay of $\left\langle c^{n}(0) c^{n}(t)\right\rangle$. At $t>0$, $\phi(t)<0$, with $\phi(t)$ climbing towards zero at large $t$. Just as $\left\langle c^{n}(0) c^{n}(t)\right\rangle$ approaches single-exponential behavior at large $n$, so also does $\phi(t)$ approach a delta function at large $n$.

In Fig. 6(a), the inset indicates the full time behavior, while the larger figure shows on a semilog scale the decay of $-\phi(t)$ out to large $t$. We looked for an analytic representation of $\phi(t)$. As seen from the early-time exponential fit (straight line), only at the shortest times is the tail of $\phi(t)$ exponential. To within calculational error, at all observed $t \phi(t)$ follows closely a two-cumulant series (solid lines)

$$
\phi(t)=a \exp \left(-K_{1} t+K_{2} t^{2} / 2\right),
$$

the $K_{i}$ being the cumulants. However, the two-cumulant series has unsatisfactory behavior in the large- $t$ limit.

Figure 6(b) gives a log-log plot of $\phi(t) / \phi(0.125)$ at $\beta J=2.5$. The decay of $\phi(t)$ depends on $n$, the decay of $\phi$ being slower for $n=2$ than for $n=0$. Data were fit both to two-cumulant series [Eq. (28)] and (for larger times only) to a power law

$$
\phi(t)=K t^{\nu} .
$$

From Fig. 6(b), the two-cumulant forms (curves) represent the measured $\phi(t)$ adequately, but become obviously unphysical at larger $t$. In contrast, long-time behavior is fit well by a power law. Higher order wavelets have a longerlived albeit weaker (note normalization in the figure) memory. For Eq. (29) we obtain $\nu=-1.8$ for $n=0$ wavelets and $\nu=-0.5$ for $n=2$ wavelets.

Figure 6(b) presents the self-consistency test for $\phi$. Equations (25) and (23) were used in separate simulations

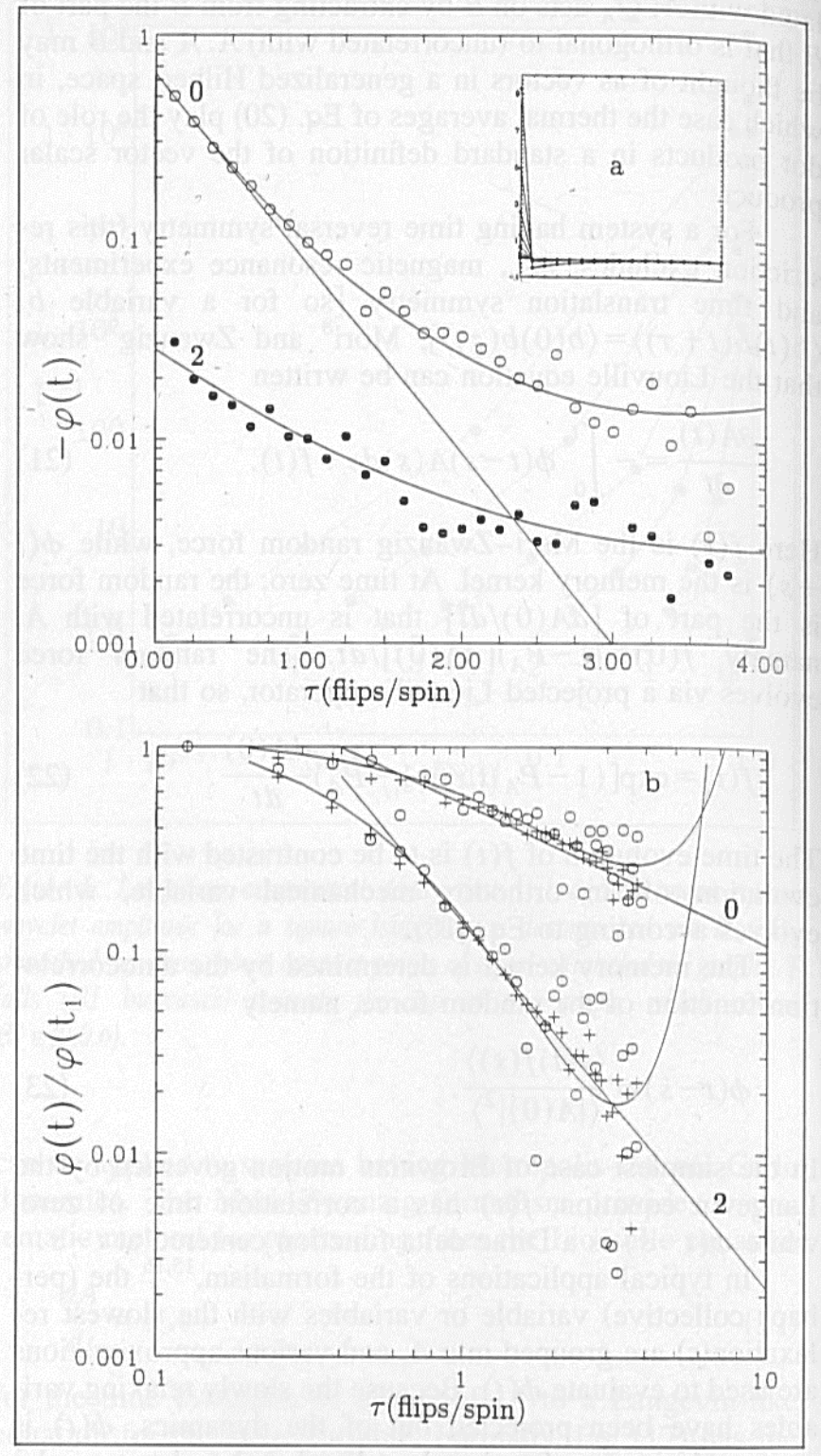

Figure 6. Memory function $\phi(t)$ for spin-spin and wavelet-wavelet correlation functions on a one-dimensional lsing ring at (a) $\beta J=0.8$ and (b) $\beta J=2.5$, using $D_{4}$ wavelets. Inset (a) shows the $t=0$ spike in $\phi(t)$; main figure shows slow recovery at longer times. In $(a), \phi(t)$ is from spin (open circles) and $n=2$ wavelet (filled circles) autocorrelation functions, and fit (lines) to initial-exponential and two-cumulant forms. Lines in (b) are two cumulant and power law fits to $\phi(t)$ computed by applying Eq. $25(+)$ or Eq. $23(\mathrm{O})$ to $n=0$ or $n=2$ wavelets (labeled 0,2 ); in the normalization of the ordinate, $t_{1}=0.125$ in natural units.

to compute $\phi_{i}$ from $\left\langle c^{n}(0) c^{n}(t)\right\rangle$ and from $f_{j}$. If $\phi_{i}$ and $f_{j}$ had been obtained by interative analyses of the same simulation run, Eq. (23) would be an identity and agreement between the two sets of points would be virtually exact. By applying Eqs. (25) and (23) to two different computer runs on the same system, an indication of the accuracy of the determination of $\phi$ for spin and wavelet dynamics is obtained. In Fig. $6(\mathrm{~b})$, pluses represent the $\phi_{i}$ computed from $\left\langle c^{n}(0) c^{n}(t)\right\rangle$, and open circles represent $\phi_{i}$ computed from $f_{j}$. The $\phi_{i}$ computed from $f_{j}$ and the fluctuationdissipation theorem is noisier than the $\phi_{i}$ from $\left\langle c^{n}(0) c^{n}(t)\right\rangle$, but there is no indication of any systematic 


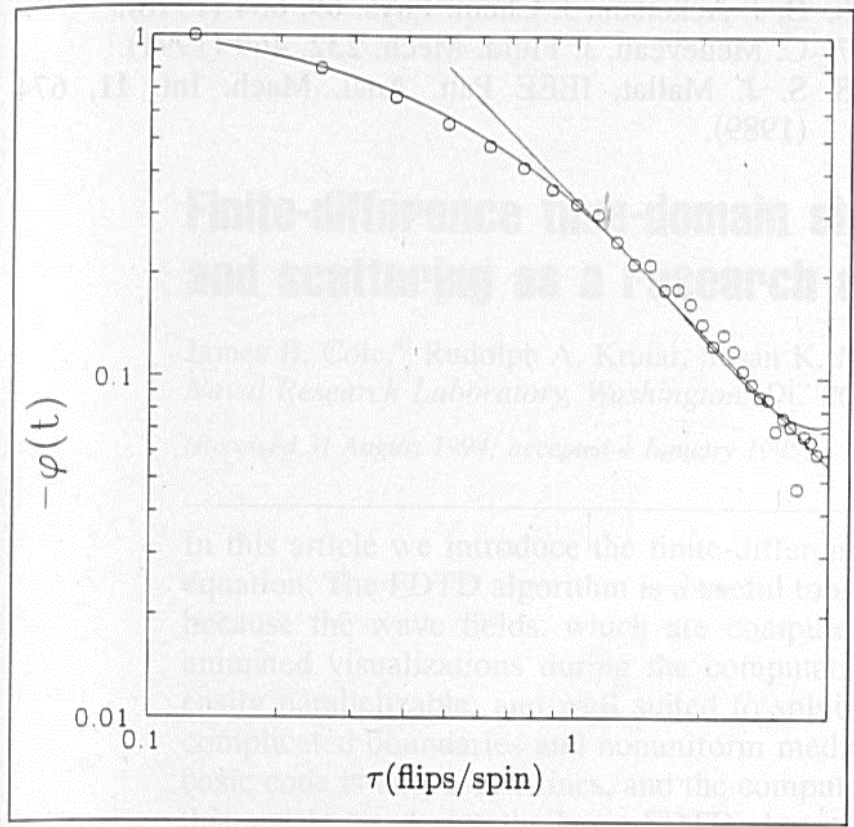

Figure 7. Memory function $\phi(t)$ for spin-spin correlations of a $64 \times 64$ square Ising lattice at $\beta J=0.4$. Fits are to a two-cumulant series (smooth curve) and a power law (straight line).

discrepancy between the two approaches to the memory kernel.

Figure 7 shows the memory function from simulations on two-dimensional $64 \times 64$ Ising lattices. Fluctuations were decomposed via $D_{4} \otimes D_{4}$ wavelets; we here emphasize the smoothed $\otimes$ smoothed components. As in Fig. 6, $\phi(t)$ has a positive spike at $t=0$, followed by a long time tail $\phi(t)<0$ for $t>0$. Figure 7 shows the long-time behavior of $\phi(t)$. Lines represent least-mean-square fits to a twocumulant series and to a power law in $t$. For clarity only $n=0$ data is shown. At large $t$ the cumulant series diverges; at large $t, \phi(t)$ clearly follows well a power law with $\nu=-1.3$.

\section{DISCUSSION}

In the above, we treat aspects of applying wavelet decompositions to the study of the Monte Carlo dynamics of a 1D or 2D Ising lattice. Several different wavelet bases, including the Haar, $D_{4}, D_{6}$, and $D_{10}$, were applied to the Ising ring. We have here shown that the decomposition is very nearly robust to the choice of wavelet basis. Dynamics of the one- and two-dimensional lattices were studied for a range of temperatures. The previously noted ${ }^{6}$ scaling relation [Eq. (16)] between the relaxation time $\Gamma^{-1}$ of the wavelet-wavelet temporal correlation function and the system compliance $\left\langle\left|c^{n}(t)\right|^{2}\right\rangle$ is indeed a high-temperature result that fails at lower temperatures (especially for smaller- $n$ wavelet decompositions); we had predicted this low-temperature failure previously. ${ }^{6}$

The significance of the choice of wavelet basis was examined previously by Meneveau, ${ }^{17}$ who examined numerical simulations of turbulent three-dimensional fluids for energy transfer. His data were projected and analyzed using Haar wavelets, Daubechies $D_{4}$ and $D_{8}$ wavelets, spatially confined Fourier transforms ("Fourierlets"), and Lemarie-Meyer-Battle ${ }^{18}$ wavelets. At fixed length scale (decimation level), Meneveau found essentially no effect of choice of wavelet basis. Decomposition of a given quantity to the same length scale gave essentially the same wavelet amplitudes, no matter which wavelet basis was used. Our result on the unimportance of the choice of wavelet basis, at least for the range of wavelet bases that we examined, is thus consistent with previous work ${ }^{17}$ on a very different physical problem.

Wavelet-wavelet temporal correlation functions decay nonexponentially, especially at lower temperatures and smaller $n$. We analyzed the nonexponential nature of the decay by applying a discrete-time generalization of the Mori-Zwanzig memory function formalism. ${ }^{8,9}$ The MoriZwanzig memory kernel $\phi(t)$ was extracted from the correlation functions $\left\langle c^{n}(0) c^{n}(t)\right\rangle$. If one specifies $\phi(t)$, the random component $f(t)$ of the force can be calculated from a second simulation of the same system; a second $\phi(t)$ can then be computed from $f(t)$ through Eq. (23). Agreement between the two $\phi(t)$ forms is a self-consistency check on the memory kernel. We made this test; as seen in Fig. 6(b), there is excellent agreement between the two determinations of $\phi(t)$, precisely as would be expected if the determination of $\phi(t)$ was physically valid.

Even for the very simple physical systems that we treat here, the Mori-Zwanzig memory kernel can have a relatively complex form. While $\phi(t)$ decays nearly exponentially at very early times, at larger $t$ the decay is quite nonexponential. Indeed, for a two-dimensional system, $\phi(t)$ corresponding to the lowest-order wavelet decimation (spin-spin correlations) has a long-time decay consistent with power-law behavior. This long-time tail in $\phi(t)$ appears likely to arise via the coupling of individual wavelets to wavelet components of longer length scales.

\section{ACKNOWLEDGMENTS}

One of us (G.P.) would like to thank Professor H. Gould, Clark University, for calling the wavelet literature to his attention. The partial support of this work by the National Science Foundation under Grant No. CHE91-15637 is gratefully acknowledged.

\section{REFEREMCES}

1. E. Ising, Z. Physik 31, 253 (1925).

2. G. Strang, SIAM Rev. 31, 614 (1989).

3. I. Daubechies, Comm. Pure Appl. Math. 41, 909 (1988).

4. C. K. Chui, Wavelets: A Tutorial in Theory and Applications (Academic, Boston, 1992).

5. G. Kaiser, A Friendly Guide to Wavelets (Birkhauser, Boston, 1994).

6. G. D. J. Phillies and J. Stott, Comput. Phys. 9, 97 (1995).

7. P. J. Burt and E. H. Adelson, ACM. Trans. Graph. 2, 217 (1983); IEEE Trans. Commun. 31, 532 (1983).

8. H. Mori, Progr. Theor. Phys. (Kyoto) 33, 432 (1965); 34, 399 (1965).

9. R. Zwanzig, J. Chem. Phys. 33, 1338 (1960).

10. D. A. McQuarrie, Statistical Mechanics (Harper and Row, New York, 1976).

11. B. J. Berne and R. Pecora, Dynamic Light Scattering (Wiley, New York, 1976).

12. W. Press, Numerical Recipes in $C, 2$ nd ed. (Cambridge University Press, New York, 1992).

13. R. J. Glauber, J. Math. Phys. 4, 294 (1963).

14. N. Metropolis, A. W. Rosenbluth, M. N. Rosenbluth, A. 
H. Teller, and E. Teller, J. Chem. Phys. 21, 1087 (1953).

15. J. P. Boon and S. Yip, Molecular Hydrodynamics (Dover, Mineola, NY, 1991).
16. B. J. Ackerson, J. Chem. Phys. 69, 684 (1978).

17. C. Meneveau, J. Fluid. Mech. 232, 469 (1991).

18. S. J. Mallat, IEEE Patt. Anal. Mach. Int. 11, 674 (1989). 